\title{
PERCEPCIONES DE ESTUDIANTES SOBRE LA TUTORIZACIÓN ACADÉMICA DEL PROFESORADO Y EL MAESTRO PSICOMOTRICISTA EN LA ADQUISICIÓN DE COMPETENCIAS PROFESIONALES
}

Perceptions of students about tutoring of lecturer and psychomotricity teacher in the acquisition of psychomotor professional skills

Perceções dos alunos sobre a tutoria académica dos professores e o professor de psicomotricidade na aquisição de competências profissionais

\section{Carolina Nieva Boza (1)}

Lurdes Martínez Mínguez (2)

Esta comunicación forma parte del siguiente proyecto de investigación competitivo: Autoevaluación de competencias de los estudiantes y desarrollo professional de maestros. Mejora de la pràctica e innovación docente trabajando por proyectos de aprendizaje psicomotores co-tutorados universidadescuela. Convocatoria de Ayudas de Investigación para la Mejora de la Formación Inicial de Maestros (ARMIF) de AGAUR. Referencia: 2017-ARMIF-00013, https://tauler.seu.cat/pagDetall.do?idEdicte $=160897 \&$ idens $=1$

(1) Universitat Autònoma de Barcelona, España. Teléfono: +34 935812674. Correo electrónico: carolina.nieva@uab.cat

(2) Universitat Autònoma de Barcelona, España. Teléfono: +34 935812674. Correo electrónico: lurdes.martinez@uab.cat

\section{Resumen}

La presente comunicación presenta una investigación llevada a cabo en la asignatura "Educación Psicomotriz en los centros de Educación Infantil", del Grado de Educación Infantil de la Universidad Autónoma de Barcelona (UAB), donde se ha realizado un Proyecto de Aprendizaje Tutorizado (PAT). Dicho estudio tiene como objetivo analizar las percepciones de los estudiantes respecto al acompañamiento compartido entre profesorado universitario y maestros psicomotricista de escuela, en la adquisición de sus competencias profesionales psicomotrices, durante su formación inicial. Los resultados más significativos se refieren a la importancia de un acompañamiento de calidad, implicación y dedicación por parte del profesorado y maestro-psicomotricista. Las conclusiones muestran que la tutorización del docente es una herramienta clave del PAT para adquisición de las competencias profesionales psicomotrices por el alumnado.

Percepciones de estudiantes sobre la tutorización académica del profesorado y el maestro psicomotricista 
Palabras clave: Proyecto de aprendizaje tutorado; tutoría; educación superior; competencias profesionales; escala de autovaloración.

\begin{abstract}
This paper explains a research carried out in the subject "Psychomotor Education in preschool education centers", of Pre-school Education Degree of the Autonomous University of Barcelona (UAB), where is carried out a Project of Oriented Learning (POL), connecting university, schools and students. This research aims to analyse the perceptions of students about tutoring shared between lecturer and psychomotricity teacher, in the acquisition of psychomotor professional skills, in the initial teacher education. The most significant results refer to the importance of a quality accompaniment, involvement and dedication on the part of the lecturer and teacher and psychomotricity teacher. The conclusions of the research show that teacher tutoring is a key tool of POL for the acquisition of psychomotor professional skills of students.
\end{abstract}

Keywords: Project of oriented learning (pol); tutoring; higher education; professional skills; auto-assessment scale

\title{
Resumo
}

A presente comunicação apresenta uma investigação realizada na disciplina "educação psicomotora nos centros de educação pré-escolar", da Licenciatura de educação préescolar da Universidade Autónoma de Barcelona (UAB), onde se realizou um projeto de Aprendizagem tutorado (PAT). O objetivo deste estudo era analisar as perceções dos alunos sobre o acompanhamento duplo de professores universitários e professores de psicomotricidade da escola, na aquisição das suas habilidades psicomotoras profissionais durante a sua formação inicial como professor. Os resultados mais significativos referem-se à importância de um acompanhamento de qualidade, envolvimento e dedicação por parte dos professores e professor de psicomotricidade. As conclusões mostram que a tutoria do professor é uma ferramenta chave do PAT para a aquisição das competências psicomotoras profissionais dos alunos.

Palavras-chave: Projeto de aprendizagem tutorado; Tutoria; Ensino superior; Competências profissionais. Escala de Autovaloração 


\section{Introducción}

El profesorado universitario tiene un papel relevante en la realización de un adecuado seguimiento personal y académico del alumnado. Según Castaño, Blanco y Asencio (2012) la acción tutorial es considerada como uno de los pilares básicos de la docencia universitaria ya que favorece el acompañamiento de su propio proceso de aprendizaje. La creación del Espacio Europeo de Educación Superior (EEES) ha representado una transformación organizativa y curricular del sistema universitario, donde las competencias se convierten en los logros de aprendizaje (Bolívar, 2008), influyendo en los objetivos, el rol del profesorado y su metodología.

La presente investigación se centra en las competencias específicas ya que están orientadas hacia experiencias que aportan sentido, globalidad y responsabilidad al alumnado (Tejada, 2012).

Para una buena adquisición de dichas competencias específicas, el profesorado debe utilizar metodologías activas favorecedoras de un aprendizaje significativo. Los Proyectos de Aprendizaje Tutorados (PAT) es una propuesta de trabajo en grupo donde se adquiere de forma autónoma un aprendizaje, desarrollando sus competencias y destrezas en diferentes contextos, y bajo la supervisión del profesorado (Álvarez, García, Gil \& Romero, 2004).

La siguiente investigación presenta un PAT dirigido por el profesorado universitario y compartido con docentes, donde nos preguntamos ¿la participación de maestros de escuela en la tutorización del PAT puede favorecer en la mejora de la adquisición de las competencias del alumnado?

Para resolver esta pregunta se plantea el siguiente objetivo: analizar las percepciones de los estudiantes en función del acompañamiento compartido entre profesorado universitario y maestros psicomotricistas de escuela, en la adquisición de sus competencias profesionales psicomotrices, en la formación inicial del maestro.

\section{Método}

Este trabajo se enmarca en el paradigma interpretativo, conocido por basarse en la búsqueda de conocimientos sobre realidades y experiencias concretas y específicas (Quintanal \& García, 2012). 
La muestra está formada por 146 estudiantes de dos asignaturas de "Educación Psicomotriz en los centros de Educación Infantil" de $4^{\circ}$ curso del Grado de Educación Infantil, de la UAB.

El instrumento que se ha utilizado ha estado la Escala de Autovaloración de Adquisición de Competencias Profesionales Psicomotrices de Educación Física, la cual ha sido contestada al inicio y al final de la asignatura. Está formada por 11 preguntas, 10 de ellas se responden a partir de la escala Likert de 1 a 6 (1 significa "muy poco", 6 significa "mucho") y la 10 va de 0 a 10.

Este estudio se ha centrado en analizar dos preguntas de la escala de autoevaluación (la $8^{\mathrm{a}}$ y la $9^{\mathrm{a}}$ ) relacionadas con el acompañamiento del profesorado y maestro.

\section{Resultados y discusión}

La Tabla 1 muestra el análisis cuantitativo de las dos preguntas:

Tabla 1.

Valoración del estudiante en función del acompañamiento recibido

\begin{tabular}{llllll}
\hline Pregunta de la escala de autovaloración & & Media & Desv. típ & Varianza \\
\hline $\begin{array}{l}\text { 8.- Valoración del profesorado respecto al grado de } \\
\text { acompañamiento que ha hecho para que adquirieses las }\end{array}$ & &, 67 &, 33 \\
$\begin{array}{l}\text { competencias profesionales psicomotrices } \\
\text { 9.- Valoración del maestro-psicomotricista de escuela respecto al } \\
\text { grado de acompañamiento que ha hecho para que adquirieses las } \\
\text { competencias profesionales psicomotrices }\end{array}$ & &, 57 &, 45 \\
\hline
\end{tabular}

Los estudiantes están bastante satisfechos con el acompañamiento recibido por ambos docentes, para la adquisición de sus competencias profesionales psicomotrices $(5,61 \mathrm{y}$ 5,67 sobre 6). Como se percibe en la tabla 2 , el grado 6 ha sido el que ha tenido una mayor frecuencia de respuestas (102 y 105 sobre 146 estudiantes).

Tabla 2.

Valoración del estudiante respecto al grado de acompañamiento mediante la escala Likert

\begin{tabular}{|c|c|c|c|c|c|c|c|}
\hline $\begin{array}{l}\text { Preguntas de la } \\
\text { escala de } \\
\text { autovaloración }\end{array}$ & $\begin{array}{c}\text { Grado } \\
1\end{array}$ & $\begin{array}{c}\text { Grado } \\
2\end{array}$ & $\begin{array}{c}\text { Grado } \\
3\end{array}$ & $\begin{array}{c}\text { Grado } \\
4\end{array}$ & $\begin{array}{c}\text { Grado } \\
5\end{array}$ & Grado 6 & $\begin{array}{l}\text { Total } \\
\text { respuestas }\end{array}$ \\
\hline $\begin{array}{l}\text { Pregunta 8: frecuencia } \\
\text { de respuestas sobre el } \\
\text { profesorado }\end{array}$ & $\begin{array}{c}0 \\
0 \%\end{array}$ & $\begin{array}{c}1 \\
, 7 \%\end{array}$ & $\begin{array}{c}1 \\
, 7 \%\end{array}$ & $\begin{array}{c}7 \\
40,7 \%\end{array}$ & $\begin{array}{c}35 \\
24 \%\end{array}$ & $\begin{array}{l}102 \\
69 \%\end{array}$ & $\begin{array}{l}146 \\
100 \%\end{array}$ \\
\hline $\begin{array}{l}\text { Pregunta 9: frecuencia } \\
\text { de respuestas sobre el } \\
\text { maestro- } \\
\text { psicomotricista }\end{array}$ & $\begin{array}{c}0 \\
0 \%\end{array}$ & $\begin{array}{c}0 \\
0 \%\end{array}$ & $\begin{array}{c}1 \\
, 7 \%\end{array}$ & $\begin{array}{c}5 \\
3,4 \%\end{array}$ & $\begin{array}{c}35 \\
24 \%\end{array}$ & $\begin{array}{c}105 \\
71,9 \%\end{array}$ & $\begin{array}{l}146 \\
100 \%\end{array}$ \\
\hline
\end{tabular}

Percepciones de estudiantes sobre la tutorización académica del profesorado y el maestro psicomotricista 
Referente a los resultados cualitativos de estas dos preguntas, se han encontrado 4 categorías (tabla 3):

Tabla 3.

Dimensiones y categorías de la investigación

\begin{tabular}{lll}
\hline \multicolumn{1}{c}{ Dimensión } & \multicolumn{1}{c}{ Categorías } \\
\hline & & 1.Relacionado con su proceso de aprendizaje \\
Acompañamiento del profesorado y el maestro & 2.Características del acompañamiento \\
$\begin{array}{l}\text { psicomotricista para la adquisición de las } \\
\text { competencias psicomotrices del alumnado }\end{array}$ & & 3.El tiempo destinado al acompañamiento \\
& & 4. Actitudes intrapersonales
\end{tabular}

Relacionado con su proceso de aprendizaje, las estudiantes perciben que la tutoría recibida tanto por el profesorado como por el maestro-psicomotricista ha sido muy favorable para la mejora de sus competencias profesionales psicomotrices.

En concreto, el profesorado les ha permitido adquirir un grado de aprendizaje mayor ya que les ha presentado un marco teórico, les ha dejado tiempo para reflexionar y les ha ofrecido estrategias para seguir avanzando en su proceso. Además, este acompañamiento les ha permitido ser más conscientes de dónde tienen más dificultades para poder superarlas.

"El acompañamiento ha sido buenísimo, sus propuestas nos orientaban y nos hacían tener otra mirada de nuestra propia propuesta. (Estudiante 63)

En la segunda categoría sobre las características del acompañamiento, los estudiantes valoran positivamente el acompañamiento recibido tanto por el profesorado como por los maestros. En concreto, perciben al profesorado como personas atentas, disponibles, empáticas, flexibles, cercanas, vocacionales y de una gran ayuda para la adquisición de las competencias.

Las estudiantes son conscientes de la gran implicación que han realizado las profesoras y destacan que este acompañamiento de calidad, dedicación e implicación hacia las estudiantes no es lo habitual en la universidad.

Referente al tiempo destinado al acompañamiento, las estudiantes lo valoran positivamente debido a la implicación de las profesoras, aunque reconocen que es una asignatura corta en tiempo para la carga tan elevada de trabajo que tiene.

Percepciones de estudiantes sobre la tutorización académica del profesorado y el maestro psicomotricista 
Por último, respecto a las actitudes intrapersonales, las estudiantes coinciden en que el factor emocional se ha cuidado durante el acompañamiento por parte de profesores y maestros. Ellas se han sentido cómodas y han recibido ánimos y energía en los momentos más difíciles del proceso.

\section{Conclusiones}

Las conclusiones de este estudio afirman que el acompañamiento compartido entre el profesorado universitario y los maestros psicomotricistas es una herramienta clave para la adquisición de las competencias profesionales psicomotrices en el grado de educación infantil. No obstante, esta tutorización debe caracterizarse por ser de calidad, con un docente que se implique y que le dedique mucho tiempo a la asignatura.

Esta experiencia educativa es un buen ejemplo de unión de universidad y escuela que puede ser transferible a otras asignaturas de la formación inicial. Sin embargo, la coherencia entre teoría y práctica debe ser un requisito básico para poder conseguir un aprendizaje competencial y vivencial.

\section{Referencias}

Álvarez Rojo, V., García Jiménez, E., Gil Flores, J., \& Romero Rodríguez, S. (2004). La enseñanza universitaria: Planificación y desarrollo de la docencia. Almería: EOS.

Bolívar, A. (2008). El discurso de las competencias en España: educación básica y educación superior. REDU. Revista de Docencia Universitaria, (2). Recuperado de http://www.redu.um.es/Red_U/m2/

Castaño, E., Blanco, A. \& Asensio, E. (2012). Competencias para la tutoría: experiencias de formación con profesorado universitario. Revista de Docencia Universitaria, $\quad 10(2), \quad 193-210 . \quad$ Recuperado de file://C:/Users/lurdes/Downloads/385-1286-1-PB.pdf

Forcadell, X. (2014). L'avaluació de les competències del psicomotricista en l'àmbit educatiu per millorar el seu desenvolupament psofessional. Universitat Autònoma de Barcelona. Departament de Didáctica de l'Expressió Musical, $\begin{array}{lllll}\text { Plàstica } & i & \text { Corporal (Tesis doctoral). Recuperado de }\end{array}$ http://www.tdx.cat/handle/10803/285371 
Quintanal, J. \& García, B. (Coords.) (2012). Fundamentos básicos de metodología de investigación educativa. Madrid: CCS.

Tejada, J. (2012). La alternancia de contextos para la adquisición de competencias profesionales en escenarios complementarios de educación superior: marco y estrategia. Educación $\quad$ XX1, 15(2), 17-40. doi: http://dx.doi.org/10.5944/educxx1.15.2.125 